\title{
SOME NEW MULTI-CELL RAMSEY THEORETIC RESULTS
}

\author{
VITALY BERGELSON AND NEIL HINDMAN
}

(Communicated by Isabella Novik)

\begin{abstract}
We extend an old Ramsey Theoretic result which guarantees sums of terms from all partition regular linear systems in one cell of a partition of the set $\mathbb{N}$ of positive integers. We were motivated by a quite recent result which guarantees a sequence in one set with all of its sums two or more at a time in the complement of that set. A simple instance of our new results is the following. Let $\mathcal{P}_{f}(\mathbb{N})$ be the set of finite nonempty subsets of $\mathbb{N}$. Given any finite partition $\mathcal{R}$ of $\mathbb{N}$, there exist $B_{1}, B_{2}, A_{1,2}$, and $A_{2,1}$ in $\mathcal{R}$ and sequences $\left\langle x_{1, n}\right\rangle_{n=1}^{\infty}$ and $\left\langle x_{2, n}\right\rangle_{n=1}^{\infty}$ in $\mathbb{N}$ such that (1) for each $F \in \mathcal{P}_{f}(\mathbb{N}), \sum_{t \in F} x_{1, t} \in B_{1}$ and $\sum_{t \in F} x_{2, t} \in B_{2}$ and (2) whenever $F, G \in \mathcal{P}_{f}(\mathbb{N})$ and $\max F<\min G$, one has $\sum_{t \in F} x_{1, t}+\sum_{t \in G} x_{2, t} \in A_{1,2}$ and $\sum_{t \in F} x_{2, t}+\sum_{t \in G} x_{1, t} \in A_{2,1}$. The partition $\mathcal{R}$ can be refined so that the cells $B_{1}, B_{2}, A_{1,2}$, and $A_{2,1}$ must be pairwise disjoint.
\end{abstract}

\section{INTRODUCTION}

Nearly half a century ago, Theorem 1.1 was proved in [7. In that theorem, $F S\left(\left\langle x_{n}\right\rangle_{n=1}^{\infty}\right)=\left\{\sum_{t \in F} x_{t}: F \in \mathcal{P}_{f}(\mathbb{N})\right\}$.

Theorem 1.1. Let $\mathcal{R}$ be a finite partition of $\mathbb{N}$. There exist $R \in \mathcal{R}$ and a sequence $\left\langle x_{n}\right\rangle_{n=1}^{\infty}$ in $\mathbb{N}$ such that $F S\left(\left\langle x_{n}\right\rangle_{n=1}^{\infty}\right) \subseteq R$.

Before the proof of Theorem 1.1] it was an observation of F. Galvin that the theorem would follow easily if one knew that there is an ultrafilter $p$ on $\mathbb{N}$ such that for every $A \in p,\{x \in \mathbb{N}:-x+A \in p\} \in p$. If one views an ultrafilter as a $\{0,1\}$-valued measure on $\mathcal{P}(\mathbb{N})$, the set of all subsets of $\mathbb{N}$, then such an ultrafilter is almost translation invariant. Given such an ultrafilter $p$ and $A_{1} \in p$, one can choose $x_{1} \in A_{1} \cap\left\{x \in \mathbb{N}:-x+A_{1} \in p\right\}$, let $A_{2}=A_{1} \cap\left(-x_{1}+A_{1}\right)$, and continue.

After the proof of the theorem, Galvin learned from S. Glazer that such ultrafilters do exist. They are simply idempotents in the compact right topological semigroup $(\beta \mathbb{N},+)$ where $\beta \mathbb{N}$ is the Stone-Čech compactification of the discrete space $\mathbb{N}$ and + extends ordinary addition on $\mathbb{N}$. (We will describe the operation + on $\beta \mathbb{N}$ later.) See the notes to [8, Section 5] for more details about the history of the proofs of Theorem 1.1 .

From the point of view of Ramsey Theory, the important property of ultrafilters is that, given any finite partition $\mathcal{R}$ of $\mathbb{N}$, some member of $\mathcal{R}$ is also member of the given ultrafilter. Later it was learned that some idempotents are better than others (from the point of view of Ramsey Theory). That is, there are idempotents whose members are guaranteed to contain rich algebraic structure. See for example 22. As a very special case, there are idempotents all of whose members contain

Received by the editors August 11, 2021, and, in revised form, October 13, 2021.

2020 Mathematics Subject Classification. Primary 05D10. 
arbitrarily long arithmetic progressions. (One of the early results in Ramsey Theory is van der Waerden's Theorem [12, which guarantees that if $\mathcal{R}$ is a finite partition of $\mathbb{N}$, then one cell of $\mathcal{R}$ contains arbitrarily long arithmetic progressions. It is a consequence of van der Waerden's Theorem that there are ultrafilters on $\mathbb{N}$ all of whose members contain arbitrarily long arithmetic progressions. The first proofs that such ultrafilters exist without invoking van der Waerden's Theorem are in 1 . and $[3]$.)

The following is a consequence of the theorem of [5]. As usual, if $C$ and $D$ are subsets of $\mathbb{N}$, then $C+D=\{c+d: c \in C$ and $d \in D\}$.

Theorem 1.2. Let $\mathcal{R}$ be a finite partition of $\mathbb{N}$. There exist $B \in \mathcal{R}$ and for each $n \in \mathbb{N}$ a length $n+2$ arithmetic progression $X_{n}$ such that, whenever $F \in \mathcal{P}_{f}(\mathbb{N})$ one has $\sum_{t \in F} X_{t} \subseteq B$.

So the cell $B$ contains not only arbitrarily long arithmetic progressions, but also a choice of such a progression for each length and all finite sums choosing at most one from each of them. In fact, there are idempotents, all of whose members contain solutions to any finite partition regular system of homogeneous linear equations with rational coefficients. We will discuss these and their relation to Furstenberg's central sets in Section 3 .

One focus of our paper is precisely how rich configurations one can get in specified cells of a partition.

In [13, Theorem 1], published in 2021, Y. Zelenyuk proved that there exist distinct points $p$ and $q$ in $\beta \mathbb{N}$ such that $p+p=q=q+q=p+q=q+p$. As a consequence, the following Ramsey Theoretic result is known to hold.

Theorem 1.3. There is a set $A \subseteq \mathbb{N}$ such that, whenever $\mathcal{R}$ is a finite partition of $A$, there exist $B \in \mathcal{R}$ and a sequence $\left\langle x_{n}\right\rangle_{n=1}^{\infty}$ in $\mathbb{N} \backslash A$ such that $\left\{\sum_{t \in F} x_{t}: F \in\right.$ $\mathcal{P}_{f}(\mathbb{N})$ and $\left.|F| \geq 2\right\} \subseteq B$.

Proof. It was shown in [4, Corollary 3.5] that the existence of $A$ would follow from the existence of $p$ and $q$ as described in the paragraph above.

The second main focus of this paper is how we can guarantee sums of specified sequences to lie in different cells, depending on their algebraic origin.

We shall derive in this paper strong extensions of Theorem 1.2. A simple consequence of Corollary 3.6 is the following more general version of the result stated in the abstract.

Theorem 1.4. Let $\delta \in \mathbb{N}$ and let $\mathcal{R}$ be a finite pariition of $\mathbb{N}$. For each $(i, j) \in$ $\{1,2, \ldots, \delta\}^{2}$, there exists $A_{i, j} \in \mathcal{R}$ such that for each $i \in\{1,2, \ldots, \delta\}$ and each $n \in \mathbb{N}$ there exists a length $n+2$ arithmetic progression $X_{i, n}$ such that whenever $F \in \mathcal{P}_{f}(\mathbb{N}), k=\min F, r=\max F$, and $\varphi: F \rightarrow\{1,2, \ldots, \delta\}$, one has $\sum_{t \in F} X_{\varphi(t), t} \subseteq A_{\varphi(k), \varphi(r)}$. The partition $\mathcal{R}$ can be refined so that the sets $\left\{A_{i, j}\right.$ : $\left.(i, j) \in\{1,2, \ldots, \delta\}^{2}\right\}$ are pairwise disjoint.

We also get some results in $(\mathbb{N}, \cdot)$. Those results are not as strong as the results in $(\mathbb{N},+)$, but they are strong enough for the full multiplicative analogue of Theorem 1.4.

Theorem 1.5. Let $\delta \in \mathbb{N}$ and let $\mathcal{R}$ be a finite pariition of $\mathbb{N}$. For each $(i, j) \in$ $\{1,2, \ldots, \delta\}^{2}$, there exists $A_{i, j} \in \mathcal{R}$ such that for each $i \in\{1,2, \ldots, \delta\}$ and each 
$n \in \mathbb{N}$ there exists a length $n+2$ geometric progression $X_{i, n}$ such that whenever $F \in \mathcal{P}_{f}(\mathbb{N}), k=\min F, r=\max F$, and $\varphi: F \rightarrow\{1,2, \ldots, \delta\}$, one has $\prod_{t \in F} X_{\varphi(t), t} \subseteq A_{\varphi(k), \varphi(r)}$. The partition $\mathcal{R}$ can be refined so that the sets $\left\{A_{i, j}\right.$ : $\left.(i, j) \in\{1,2, \ldots, \delta\}^{2}\right\}$ are pairwise disjoint.

Our proofs use the algebraic structure of $\beta \mathbb{N}$. We present a brief introduction to that structure as well as some preliminary results in Section 2 .

In Section 3 we present results dealing with finite partitions of $\mathbb{N}$. These results follow from the existence of finite algebraic structures in $\beta \mathbb{N}$.

In Section 4 we present results following from the existence of infinite algebraic structures in $\beta \mathbb{N}$.

\section{Algebraic Background}

We take the Stone-Čech compactification $\beta X$ of a discrete space $X$ to consist of the ultrafilters on $X$, identifying the point $x \in X$ with the principal ultrafilter $e(x)=\{A \subseteq X: x \in A\}$. The topology on $\beta X$ has a base for the open sets (which is also a base for the closed sets) consisting of $\{\bar{A}: A \subseteq X\}$, where $\bar{A}=\{p \in \beta X$ : $A \in p\}$.

If $(S,+)$ is a discrete semigroup, the operation extends to $\beta S$ making $(\beta S,+)$ a compact right topological semigroup (meaning that the function $\rho_{p}$ defined by $\rho_{p}(q)=q+p$ is continuous for each $p \in \beta S$ ) with $S$ contained in its topological center (meaning that the function $\lambda_{x}$ defined by $\lambda_{x}(q)=x+q$ is continuous for each $x \in S$ ). One can also extend the operation so that $\lambda_{p}$ is continuous for each $p \in \beta S$ and $\rho_{x}$ is continuous for each $x \in S$. In that case, $(\beta S,+)$ is a left topological semigroup. The reader should be warned that in [2] and [3] we took $(\beta S,+)$ to be left topological.

If $p, q \in \beta S$ and $A \subseteq S$, then $A \in p+q$ if and only if $\{x \in S:-x+A \in q\} \in p$ where $-x+A=\{y \in S: x+y \in A\}$. If the operation is denoted by - we write $A \in p \cdot q$ if and only if $\left\{x \in S: x^{-1} A \in q\right\} \in p$ where $x^{-1} A=\{y \in S: x \cdot y \in A\}$.

As does any compact Hausdorff right topological semigroup, $(\beta S,+)$ has a smallest two sided ideal, $K(\beta S)$, which is the union of all of the minimal left ideals and is also the union of all of the minimal left ideals. If $L$ is a minimal left ideal and $R$ is a minimal right ideal, then $L \cap R$ is a group. Note that if $L$ is a minimal left ideal of $\beta S$ and $p \in L$, then $L=\beta S+p=\rho_{p}[\beta S]$ so as the continuous image of a compact space, $L$ is compact. On the other hand, minimal right ideals are usually not compact. For an elementary introduction to the algebraic structure of $\beta S$, see [8. Part I].

Of particular interest for us in this paper are rectangular semigroups.

Definition 2.1. A rectangular semigroup $R$ is the Cartesian product of a left zero semigroup $A$ with a right zero semigroup $B$. That is, $R=A \times B$ and given $(a, b)$ and $(c, d)$ in $R,(a, b) \cdot(c, d)=(a, d)$.

Note that the algebra of $R$ is completely determined by $|A|$ and $|B|$, so we will refer to "the $|A| \times|B|$ rectangular semigroup". Note also that if $R$ is a rectangular semigroup, and $(a, b),(c, d),(e, f) \in R$, then $(a, b) \cdot(c, d) \cdot(e, f)=(a, b) \cdot(e, f)$.

Theorem 2.2. There is an algebraic copy of the $2^{\mathfrak{c}} \times 2^{\mathfrak{c}}$ rectangular semigroup contained in $K(\beta \mathbb{N},+)$. There is also an algebraic copy of the $2^{\mathfrak{c}} \times 2^{\mathfrak{c}}$ rectangular semigroup contained in $K(\beta \mathbb{N}, \cdot)$. 
Proof. The first assertion is an immediate consequence of [9, Corollary 3.15]. Let $\mathbb{H}=\bigcap_{n=1}^{\infty} \overline{2^{n} \mathbb{N}}$. By [8, Lemma 6.8], $\mathbb{H}$ is a compact subsemigroup of $(\beta \mathbb{N},+)$ which contains all of the idempotents. Thus by [8, Theorem 1.65], $K(\mathbb{H})=K(\beta \mathbb{N},+) \cap \mathbb{H}$, so $K(\mathbb{H})$ contains an algebraic copy of the $2^{\mathfrak{c}} \times 2^{\mathfrak{c}}$ rectangular semigroup. By 9 , Theorem 2.4], $K(\beta \mathbb{N}, \cdot)$ contains an algebraic copy of $K(\mathbb{H})$ so the second assertion follows.

We proceed to present in Theorem 2.5]a result due to Dona Strauss in a personal communication. We recall that cardinal numbers are ordinals and each ordinal is the set of its predecessors. For example $2^{\mathfrak{c}}$ is the cardinal number of the power set of $\mathbb{R}$. As such, it is a set with the same number of members as $\mathcal{P}(\mathbb{R})$ whose members are all ordinals smaller than $\mathcal{P}(\mathbb{R})$. Also, $\omega=\mathbb{N} \cup\{0\}$ is the first infinite cardinal.

Lemma 2.3. Let $\left\langle M_{b}\right\rangle_{b \in 2^{c}}$ be a pairwise disjoint collection of compact subsets of $\beta \mathbb{N}$. There exist a sequence $\left\langle b_{n}\right\rangle_{n=1}^{\infty}$ in $2^{\mathfrak{c}}$ and a sequence $\left\langle A_{n}\right\rangle_{n=1}^{\infty}$ in $\mathcal{P}(\mathbb{N})$ such that $A_{n} \cap A_{m}=\emptyset$ if $n \neq m$ and $M_{b_{n}} \subseteq \overline{A_{n}}$ for each $n \in \mathbb{N}$.

Proof. For $A \subseteq \mathbb{N}$, let $B_{A}=\left\{d \in 2^{\mathfrak{c}}: M_{d} \cap \bar{A}=\emptyset\right\}$. Pick $b_{1} \in 2^{\mathfrak{c}}$ and let $\mathcal{A}_{1}=\left\{A \subseteq \mathbb{N}: M_{b_{1}} \subseteq \bar{A}\right\}$. We claim that $2^{\mathfrak{c}} \backslash\left\{b_{1}\right\} \subseteq \bigcup_{A \in \mathcal{A}_{1}} B_{A}$. To see this, let $d \in 2^{\mathfrak{c}} \backslash\left\{b_{1}\right\}$. Then $M_{b_{1}}$ and $M_{d}$ are disjoint compact subsets of $\beta \mathbb{N}$ so there exists $A \subseteq \mathbb{N}$ such that $M_{b_{1}} \subseteq \bar{A}$ and $M_{d} \cap \bar{A}=\emptyset$. Then $A \in \mathcal{A}_{1}$ and $d \in B_{A}$ as required. Since $\left|2^{\mathfrak{c}} \backslash\left\{b_{1}\right\}\right|=2^{\mathfrak{c}}$ and $\left|\mathcal{A}_{1}\right|=\mathfrak{c}$, we may pick $A \in \mathcal{A}_{1}$ such that $\left|B_{A_{1}}\right|=2^{\mathfrak{c}}$.

Now let $n \in \mathbb{N}$ and assume we have chosen $\left\langle b_{i}\right\rangle_{i=1}^{n}$ in $2^{\mathfrak{c}}$ and $\left\langle A_{i}\right\rangle_{i=1}^{n}$ in $\mathcal{P}(\mathbb{N})$ such that

(1) for $i \in\{1,2, \ldots, n\}, M_{b_{1}} \subseteq \overline{A_{i}}$,

(2) for $i \in\{2,3, \ldots, n\}$, if any, $b_{i} \in \bigcap_{j=1}^{i-1} B_{A_{j}}$,

(3) $\left|\bigcap_{i=1}^{n} B_{A_{i}}\right|=2^{\mathfrak{c}}$, and

(4) if $i \neq j$ in $\{1,2, \ldots, n\}$, then $A_{i} \cap A_{j}=\emptyset$.

Pick $b_{n+1} \in \bigcap_{i=1}^{n} B_{A_{i}} \backslash\left\{b_{1}, b_{2}, \ldots, b_{n}\right\}$. Let

$$
\mathcal{A}_{n+1}=\left\{A \subseteq \mathbb{N}: A \cap \bigcup_{i=1}^{n} A_{i}=\emptyset \text { and } M_{b_{n+1}} \subseteq \bar{A}\right\} .
$$

We claim that $\bigcap_{i=1}^{n} B_{A_{i}} \backslash\left\{b_{1}, b_{2}, \ldots, b_{n+1}\right\} \subseteq \bigcup_{A \in \mathcal{A}_{n+1}}\left(B_{A} \cap \bigcap_{i=1}^{n} B_{A_{i}}\right)$. To see this, let $d \in \bigcap_{i=1}^{n} B_{A_{i}} \backslash\left\{b_{1}, b_{2}, \ldots, b_{n+1}\right\}$. Since $b_{n+1} \in \bigcap_{i=1}^{n} B_{A_{i}}, M_{b_{n+1}} \cap \overline{\bigcup_{i=1}^{n} A_{i}}=\emptyset$. Since $d \neq b_{n+1}, M_{b_{n+1}} \cap M_{d}=\emptyset$. Thus $M_{b_{n+1}}$ and $M_{d} \cup \overline{\bigcup_{i=1}^{n} A_{i}}$ are disjoint compact subsets of $\beta \mathbb{N}$ so we may pick $A \subseteq \mathbb{N}$ such that $M_{b_{n+1}} \subseteq \bar{A}$ and $\bar{A} \cap$ $\left(M_{d} \cup \overline{\bigcup_{i=1}^{n} A_{i}}\right)=\emptyset$. Then $A \in \mathcal{A}_{n+1}$ and $d \in B_{A} \cap \bigcap_{i=1}^{n} B_{A_{i}}$ as claimed. Since $\left|\bigcap_{i=1}^{n} B_{A_{i}} \backslash\left\{b_{1}, b_{2}, \ldots, b_{n+1}\right\}\right|=2^{\mathfrak{c}}$ and $\left|\mathcal{A}_{n+1}\right| \leq \mathfrak{c}$, we may pick $A_{n+1} \in \mathcal{A}_{n+1}$ such that $\left|B_{A_{n+1}} \cap \bigcap_{i=1}^{n} B_{A_{i}}\right|=2^{\mathfrak{c}}$. All hypotheses are satisfied.

Lemma 2.4. Let $R$ be a minimal right ideal of $\beta \mathbb{N}$ and let $E(R)$ be the set of idempotents in $R$. Then every member of $c \ell(E(R))$ is a left identity for $R$.

Proof. If $p \in E(R)$, then $R=p+\beta \mathbb{N}$ so if $r \in R$, then $r=p+q$ for some $q$ so $p+r=p+p+q=p+q=r$. Now let $p \in c \ell(E(R))$ and suppose we have some $x \in R$ such that $p+x \neq x$. Pick $A \in x \backslash(p+x)$. Then $\mathbb{N} \backslash A \in p+x$ so pick $B \in p$ such that $\bar{B}+x \subseteq \overline{\mathbb{N} \backslash A}$. Pick $q \in \bar{B} \cap E(R)$. Then $x=q+x$ so $A \cap(\mathbb{N} \backslash A) \neq \emptyset$, a contradiction. 
Theorem 2.5. There is a discrete copy of the $\omega \times \omega$ rectangular semigroup in $K(\beta \mathbb{N},+)$.

Proof. By Theorem 2.2 pick an injective function $\varphi: 2^{\mathfrak{c}} \times 2^{\mathfrak{c}} \rightarrow K(\beta \mathbb{N},+)$ such that for $(a, b),(c, d) \in 2^{\mathfrak{c}} \times 2^{\mathfrak{c}}, \varphi(a, b)+\varphi(c, d)=\varphi(a, d)$. For $(a, b) \in 2^{\mathfrak{c}} \times 2^{\mathfrak{c}}$, let $p_{a, b}=\varphi(a, b)$. We now claim that for each $a \in 2^{\mathfrak{c}}$, there exist a minimal left ideal $L_{a}$ of $\beta \mathbb{N}$ and a minimal right ideal $R_{a}$ of $\beta \mathbb{N}$ such that for $(a, b) \in 2^{\mathfrak{c}} \times 2^{\mathfrak{c}}, p_{a, b}$ is the identity of $R_{a} \cap L_{b}$. To see this, let $(a, b) \in 2^{\mathfrak{c}} \times 2^{\mathfrak{c}}$ and let $L_{b}$ be the minimal left ideal of $\beta \mathbb{N}$ in which $p_{a, b}$ lies. Then, given any $c \in 2^{\mathfrak{c}}, p_{c, b}=p_{c, b}+p_{a, b} \in L_{b}$. Similarly, if $R_{a}$ is the minimal right ideal in which $p_{a, b}$ lies, then for any $c \in 2^{\mathfrak{c}}$, $p_{a, c}=p_{a, b}+p_{a, c} \in R_{a}$.

We have for $b \in 2^{\mathfrak{c}}$ that $L_{b}$ is compact and $L_{b} \cap L_{c}=\emptyset$ if $b \neq c$. By Lemma 2.3pick a sequence $\left\langle b_{n}\right\rangle_{n=0}^{\infty}$ in $2^{\mathfrak{c}}$ and a sequence $\left\langle A_{n}\right\rangle_{n=0}^{\infty}$ in $\mathcal{P}(\mathbb{N})$ such that $A_{n} \cap A_{m}=\emptyset$ if $n \neq m$ and $L_{b_{n}} \subseteq \overline{A_{n}}$ for each $n \in \omega$.

For each $c \in 2^{\mathfrak{c}}$, let $D_{c}=\left\{p_{c, b_{n}}: n \in \omega\right\}$. We claim that if $c$ and $d$ are distinct members of $2^{\mathfrak{c}}$, then $c \ell D_{c} \cap c \ell D_{d}=\emptyset$. So suppose instead we have $c \ell D_{c} \cap c \ell D_{d} \neq \emptyset$. Then by [8, Theorem 3.40] either there is some $n \in \omega$ such that $p_{c, b_{n}} \in c \ell D_{d}$ or there is some $n \in \omega$ such that $p_{d, b_{n}} \in c \ell D_{c}$. We assume without loss of generality the former. Since $D_{d} \subseteq E\left(R_{d}\right)$, we have by Lemma 2.4 that $p_{c, b_{n}}$ is a left identity for $R_{d}$. But $p_{c, b_{n}}+p_{d, b_{n}}=p_{c, b_{n}} \notin R_{d}$, a contradiction.

By Lemma 2.3 pick a sequence $\left\langle c_{n}\right\rangle_{n=0}^{\infty}$ in $2^{\mathfrak{c}}$ and a sequence $\left\langle C_{n}\right\rangle_{n=0}^{\infty}$ in $\mathcal{P}(\mathbb{N})$ such that $C_{n} \cap C_{m}=\emptyset$ if $n \neq m$ and $c \ell D_{c_{n}} \subseteq \overline{C_{n}}$ for each $n \in \omega$.

Then $R=\left\{p_{c_{n}, b_{m}}: n, m \in \omega\right\}$ is an algebraic copy of the $\omega \times \omega$ rectangular semigroup and given $(n, m) \in \omega \times \omega, R \cap \overline{C_{n}} \cap \overline{A_{m}}=\left\{p_{c_{n}, b_{m}}\right\}$.

Notice that the proof actually shows that the copy of the $\omega \times \omega$ rectangular semigroup is strongly discrete. That is, if $(n, m) \neq(k, l)$, then $\left(\overline{C_{n}} \cap \overline{A_{m}}\right) \cap\left(\overline{C_{k}} \cap\right.$ $\left.\overline{A_{l}}\right)=\emptyset$.

Corollary 2.6. There exists a sequence $\left\langle p_{n}\right\rangle_{n=1}^{\infty}$ of distinct idempotents in $K(\beta \mathbb{N})$ such that for all $n, m \in \mathbb{N}, p_{n}+p_{m}$ is and idempotent and if $k, n, m \in \mathbb{N}$, then $p_{n}+p_{k}+p_{m}=p_{n}+p_{m}$. Further, if $(n, m) \neq(k, l)$, then $p_{n}+p_{m} \neq p_{m}+p_{n}$ and $\left\{p_{n}: n \in \mathbb{N}\right\} \cup\left\{p_{n}+p_{m}: n \neq m\right.$ in $\left.\mathbb{N}\right\}$ is strongly discrete.

Proof. Let $\left\{p_{n, m}: n, m \in \omega\right\}$ be a copy of the $\omega \times \omega$ rectangular semigroup as guaranteed by Theorem 2.5. For $n \in \mathbb{N}$, let $p_{n}=p_{n, n}$.

\section{Resuts about finite partitions}

We begin this section with a very special case of our main finite results. We do so because the proof already contains the main ideas needed for the more general proofs without the additional complications. For this result we do not need to assume that the idempotents are in $K(\beta \mathbb{N})$.

If $q=q+q$ in $\beta \mathbb{N}$ and $C \in q$, then $C^{\star}(q)=\{x \in C:-x+C \in q\}$. By [8, Lemma 4.14], if $C \in q$ and $x \in C^{\star}(q)$, then $-x+C^{\star}(q) \in q$. In our proofs we will write $C^{\star}$ rather than $C^{\star}(q)$, relying on the context to determine the relevant idempotent.

The referee noted that the existence of the sequences produced in Theorem 3.1 can also be deduced from [14, Theorem 4.2]. The referee also observed that $\left\{p_{h}+p_{i}:(h, i) \in\{1,2, \ldots, \delta\}^{2}\right\}$ in this theorem forms a rectangular semigroup. 
Theorem 3.1. Let $\delta \in \mathbb{N} \backslash\{1\}$ and let $\left\langle p_{h}\right\rangle_{h=1}^{\delta}$ be a sequence of distinct idempotents in $\beta \mathbb{N}$ such that for $(h, i) \in\{1,2, \ldots, \delta\}^{2}, p_{h}+p_{i}$ is an idempotent and $\left\{p_{h}+p_{i}\right.$ : $\left.(h, i) \in\{1,2, \ldots, \delta\}^{2}\right\}$ are all distinct. Assume that for $(h, i, j) \in\{1,2, \ldots, \delta\}^{3}$, $p_{h}+p_{i}+p_{j}=p_{h}+p_{j}$. For $(h, i) \in\{1,2, \ldots, \delta\}^{2}$, let $A_{h, i} \in p_{h}+p_{i}$ such that $\left\{A_{h, i}\right.$ : $\left.(h, i) \in\{1,2, \ldots, \delta\}^{2}\right\}$ consists of pairwise disjoint sets. For each $h \in\{1,2, \ldots, \delta\}$, there exists a sequence $\left\langle x_{h, t}\right\rangle_{t=1}^{\infty}$ in $\mathbb{N}$ such that if $F \in \mathcal{P}_{f}(\mathbb{N}), k=\min F, r=$ $\max F$, and $\varphi: F \rightarrow\{1,2, \ldots, \delta\}$, then $\sum_{t \in F} x_{\varphi(t), t} \in A_{\varphi(k), \varphi(r)}$.

Proof. For $h \in\{1,2, \ldots, \delta\}$, let $B_{h, 1}=A_{h, h} \cap \bigcap_{i=1}^{\delta} \bigcap_{j=1}^{\delta}\left\{x \in \mathbb{N}:-x+A_{h, j}^{\star} \in\right.$ $\left.p_{i}+p_{j}\right\}$. Notice that for $(h, i, j) \in\{1,2, \ldots, \delta\}^{3}$, since $A_{h, j}^{\star} \in p_{h}+p_{j}=p_{h}+p_{i}+p_{j}$, $\left\{x \in \mathbb{N}:-x+A_{h, j}^{\star} \in p_{i}+p_{j}\right\} \in p_{h}$.

We shall construct inductively for $n \in \mathbb{N}$ and $h \in\{1,2, \ldots, \delta\},\left\langle B_{h, s}\right\rangle_{s=1}^{n}$ and $\left\langle x_{h, s}\right\rangle_{s=1}^{n}$ satisfying the following induction hypotheses.

(1) For $s \in\{1,2, \ldots, n\}$ and $h \in\{1,2, \ldots, \delta\}$

(a) $B_{h, s} \in p_{h}$ and if $s<n, B_{h, s+1} \subseteq B_{h, s}$ and

(b) $x_{h, s} \in B_{h, s}^{\star}$.

(2) If $\emptyset \neq F \subseteq\{1,2, \ldots, n\}, k=\min F, r=\max F$, and $\varphi: F \rightarrow\{1,2, \ldots, \delta\}$, then

(a) $\sum_{t \in F} x_{\varphi(t), t} \in A_{\varphi(k), \varphi(r)}^{\star}$;

(b) if $r<n,(b, h, d, e) \in\{1,2, \ldots, \delta\}^{4}, z=\sum_{t \in F} x_{\varphi(t), t}$, and $-z+$ $A_{\varphi(k), b}^{\star} \in p_{h}+p_{d}$, then $B_{h, r+1} \subseteq\left\{y \in \mathbb{N}:-(z+y)+A_{\varphi(k), b}^{\star} \in p_{e}+p_{d}\right\}$; and

(c) if $r<n,(b, h) \in\{1,2, \ldots, k\}^{2}, z=\sum_{t \in F} x_{\varphi(t), t}$, and $-z+A_{\varphi(k), b}^{\star} \in$ $p_{h}$, then $B_{h, r+1} \subseteq-z+A_{\varphi(k), b}^{\star}$.

We shall inductively define $B_{h, n}$, taking in each case an intersection of finitely many members of $p_{h}$.

We have already defined $B_{h, 1}$ for $h \in\{1,2, \ldots, \delta\}$; noting that $B_{h, 1} \in p_{h}$, pick $x_{h, 1} \in B_{h, 1}^{\star}$.

The hypotheses are satisfied at $n=1$. (Hypothesis (2)(a) says that $x_{\varphi(1), 1} \in$ $\left.B_{\varphi(1)}^{\star} \cdot\right)$

Now assume $n \in \mathbb{N}$ and the hypotheses hold at $n$. For $h \in\{1,2, \ldots, \delta\}$ let $B_{h, n+1}$ be the intersection of $B_{h, n}^{\star}$ with sets of the following two forms.

(i) If $\emptyset \neq F \subseteq\{1,2, \ldots, n\}, k=\min F, r=\max F=n, \varphi: F \rightarrow\{1,2, \ldots, \delta\}$, and one has $(b, d, e) \in\{1,2, \ldots, \delta\}^{3}$ and $z=\sum_{t \in F} x_{\varphi(t), t}$ such that $-z+$ $A_{\varphi(k), b}^{\star} \in p_{h}+p_{d}$, then intersect with $\left\{y \in \mathbb{N}:-(z+y)+A_{\varphi(k), b}^{\star} \in p_{e}+p_{d}\right\}$. Since $p_{h}+p_{d}=p_{h}+p_{e}+p_{d}$, these sets are in $p_{h}$.

(ii) If $\emptyset \neq F \subseteq\{1,2, \ldots, n\}, k=\min F, r=\max F=n, \varphi: F \rightarrow\{1,2, \ldots, \delta\}$, and one has $b \in\{1,2, \ldots, \delta\}$ and $z=\sum_{t \in F} x_{\varphi(t), t}$ such that $-z+A_{\varphi(k), b} \in$ $p_{h}$, then intersect with $-z+A_{\varphi(k), b}^{\star}$.

Notice that $B_{h, n+1}$ is the intersection of finitely many members of $p_{h}$. Pick $x_{h, n+1} \in B_{h, n+1}^{\star}$.

To verify hypothesis (1), let $h \in\{1,2, \ldots, \delta\}$ and let $s \in\{1,2, \ldots, n+1\}$. If $s \leq n$, then hypothesis (1) holds by assumption. If $s=n+1$, then hypothesis (1) holds by construction.

To verify hypothesis $(2)$, let $\emptyset \neq F \subseteq\{1,2, \ldots, n+1\}$, let $k=\min F$, let $r=\max F$, and let $\varphi: F \rightarrow\{1,2, \ldots, \delta\}$. If $r<n$, then hypothesis (2) holds by assumption. Assume now that $r=n$. Then hypothesis (2)(a) holds by assumption. 
To verify (2)(b), assume we have $(b, h, d, e) \in\{1,2, \ldots, \delta\}^{4}$, and $z=\sum_{t \in F} x_{\varphi(t), t}$ such that $-z+A_{\varphi(k), b}^{\star} \in p_{h}+p_{d}$. Then by item (i) in the construction, $B_{h, n+1} \subseteq$ $\left\{y \in \mathbb{N}:-(z+y)+A_{\varphi(k), b}^{\star} \in p_{e}+p_{d}\right\}$. To verify (2)(c), assume we have $(b, h) \in$ $\{1,2, \ldots, \delta\}^{2}$ and $z=\sum_{t \in F} X_{\varphi(t), t}$, such that $-z+A_{\varphi(k), b}^{\star} \in p_{h}$. Then by item (ii) in the construction, $B_{h, n+1} \subseteq-z+A_{\varphi(k), b}^{\star}$.

Now assume that $r=n+1$, so that (2)(b) and (2)(c) are vacuous. We shall verify (2)(a). Assume first that $|F|=1$, so that $F=\{n+1\}$ and $k=r=n+1$. Let $h=\varphi(n+1)$. Then $x_{\varphi(n+1), n+1} \in B_{h, n+1}^{\star} \subseteq B_{h, 1}^{\star} \subseteq A_{h, h}^{\star}$ as required.

Now assume that $|F| \geq 2$, let $G=F \backslash\{n+1\}$, and let $l=|G|$. Let $t_{1}, t_{2}, \ldots, t_{l}$ be the members of $G$ in order and let $t_{l+1}=n+1$. We shall show that

$$
\begin{aligned}
& \text { for } u \in\{1,2, \ldots, l\} \text { if } z=\sum_{s=1}^{u} x_{\varphi\left(t_{s}\right), t_{s}} \text {, then } \\
& -z+A_{\varphi(k), \varphi(r)}^{\star} \in p_{\varphi\left(t_{u+1}\right)}+p_{\varphi(r)} .
\end{aligned}
$$

For $u=1, z=x_{\varphi(k), k} \in B_{\varphi(k), 1}^{\star} \subseteq\left\{y \in \mathbb{N}:-y+A_{\varphi(k), \varphi(r)} \in p_{\varphi\left(t_{2}\right)}+p_{\varphi(r)}\right\}$.

If $l=1$, then $(*)$ has been established. Assume that $l \geq 2, u \in\{2,3, \ldots, l\}$ and if $z=\sum_{s=1}^{u-1} x_{\varphi\left(t_{s}\right), t_{s}}$, then $-z+A_{\varphi(k), \varphi(r)}^{\star} \in p_{\varphi\left(t_{u}\right)}+p_{\varphi(r)}$. Let $w=\sum_{s=1}^{u} x_{\varphi\left(t_{s}\right), t_{s}}$ and let $z=\sum_{s=1}^{u-1} x_{\varphi\left(t_{s}\right), t_{s}}$ so that $w=z+x_{\left.\varphi\left(t_{u}\right), t_{u}\right)}$. Let $v=t_{u-1}=\max \left\{t_{1}, \ldots t_{u-1}\right\}$. Then $v<n$ so by hypothesis (2)(b) with $h=\varphi\left(t_{u}\right), e=\varphi\left(t_{u+1}\right)$ and $d=b=\varphi(r)$, $-z+A_{\varphi(k), \varphi(r)} \in p_{\varphi\left(t_{u}\right)}+p_{\varphi(r)}$ so $B_{\varphi\left(t_{u}\right), v+1} \subseteq\left\{y \in \mathbb{N}:-(z+y)+A_{\varphi(k), \varphi(r)}^{\star} \in\right.$ $p_{\varphi\left(t_{u+1}\right)}+p_{\varphi(r)}$. Then $x_{\left.\varphi\left(t_{u}\right), t_{u}\right)} \in B_{\varphi\left(t_{u}\right), t_{u}}^{\star} \subseteq B_{\varphi\left(t_{u}\right), v+1}$ so $-w+A_{\varphi(k), \varphi(r)}^{\star} \in$ $p_{\varphi\left(t_{u+1}\right)}+p_{\varphi(r)}$. Thus $(*)$ has been established. In particular if $z=\sum_{s=1}^{l} x_{\varphi\left(t_{s}\right), t_{s}}$, then $-z+A_{\varphi(k), \varphi(r)}^{\star} \in p_{\varphi\left(t_{l+1}\right)}+p_{\varphi(r)}=p_{\varphi(r)}$ since $t_{l+1}=n+1=r$. That is, if $z=\sum_{t \in G} x_{\varphi(t), t}$, then $-z+A_{\varphi(k), \varphi(r)}^{\star} \in p_{\varphi(r)}$.

Let $v=\max G$. Let $w=\sum_{t \in F} x_{\varphi(t), t}$ and let $z=\sum_{t \in G} x_{\varphi(t), t}$ so that $w=$ $z+x_{\varphi(r), r}$. Then $B_{\varphi(r), v+1} \subseteq-z+A_{\varphi(k), \varphi(r)}^{\star}$ by item (ii) in the construction if $v=n$ and by hypothesis (2)(c) applied to $G$ with $b=h=\varphi(r)$ if $v<n$. Then $x \in B_{\varphi(r), v+1}$ so $w \in A_{\varphi(k), \varphi(r)}^{\star}$.

Notice in particular, taking the function $\varphi$ to be constant, that one has for each $h \in\{1,2, \ldots, \delta\}$, that $F S\left(\left\langle x_{h, t}\right\rangle_{t=1}^{\infty} \subseteq A_{h, h}\right.$.

A system of homogeneous linear equations is partition regular over $\mathbb{N}$ if and only if, whenever $\mathcal{R}$ is a finite partition of $\mathbb{N}$, there exists $R \in \mathcal{R}$ which contains a solution to the system of equations. In that case, we say that the coefficient matrix is kernel partition regular.

Definition 3.2. Let $u, v \in \mathbb{N}$ and let $M$ be a $u \times v$ matrix with rational coefficients. Then $M$ is kernel partition regular if and only if, whenever $\mathcal{R}$ is a finite partition of $\mathbb{N}$, there exist $R \in \mathcal{R}$ and $\vec{x} \in R^{v}$ such that $M \vec{x}=\overrightarrow{0}$.

R. Rado [10] characterized the kernel partition regular matrices as those that satisfy the columns condition. We will not have to use the columns condition in this paper, so we won't describe it. We will need a different characterization in terms of central sets. 
The notion of central subsets of $\mathbb{N}$ was introduced by H. Furstenberg in 6 , Chapter 8] and defined using notions from topological dynamics 1 He proved that any central subset of $\mathbb{N}$ contains solutions for any partition regular system of homogeeneous linear equations. The definition that we will use was shown to be equivalent to Furstenberg's definition for countable semigroups in [3] and for general semigroups by $\mathrm{H}$. Shi and H. Yang in [11.

Idempotents in $K(\beta S)$ are called minimal idempotents. As we noted, $K(\beta S)=$ $\bigcup\{L: L$ is a minimal left ideal of $\beta S\}=\bigcup\{R: R$ is a minimal right ideal of $\beta S\}$. So an idempotent is minimal if and only if it is a member of some minimal left ideal and also if and only if it is a member of some minimal right ideal.

Definition 3.3. Let $(S,+)$ be a semigroup and let $C \subseteq S$. Then $C$ is central if and only if $C$ is a member of a minimal idempotent of $\beta S$.

Theorem 3.4. Let $u, v \in \mathbb{N}$ and let $M$ be $a u \times v$ matrix with rational entries. Then $M$ is kernel partition regular over $\mathbb{N}$ if and only if for each central subset $C$ of $\mathbb{N}$, there exists $\vec{x} \in C^{v}$ such that $M \vec{x}=\overrightarrow{0}$.

Proof. Since one cell of any partition of $\mathbb{N}$ must be central, the sufficiency is immediate. The necessity was proved using the original (dynamic) definition in [6. Chapter 8, Section 7]. A proof using the algebraic definition is in [8, Theorem 16.14(b)].

Theorem 3.5. Let $\delta \in \mathbb{N} \backslash\{1\}$. Let $\left\langle M_{n}\right\rangle_{n=1}^{\infty}$ enumerate the finite kernel partition regular matrices with rational coefficients and for $n \in \mathbb{N}$ let $m(n)$ be the number of columns of $M_{n}$. Let $\left\langle p_{i}\right\rangle_{i=1}^{\delta}$ be a sequence of distinct idempotents in $K(\beta \mathbb{N},+)$ such that for $(i, j) \in\{1,2, \ldots, \delta\}^{2}, p_{i}+p_{j}$ is an idempotent and $\left\{p_{i}+p_{j}:(i, j) \in\right.$ $\left.\{1,2, \ldots, \delta\}^{2}\right\}$ are all distinct. Assume that for $(h, i, j) \in\{1,2, \ldots, \delta\}^{3}, p_{h}+p_{i}+$ $p_{j}=p_{h}+p_{j}$. For $(i, j) \in\{1,2, \ldots, \delta\}^{2}$, let $A_{i, j} \in p_{i}+p_{j}$. For $i \in\{1,2, \ldots, \delta\}$ and $n \in \mathbb{N}$, there exists $\vec{x}(i, n) \in \mathbb{N}^{m(n)}$ such that $M_{n} \vec{x}(i, n)=\overrightarrow{0}$ and, letting $X_{i, n}$ be the set of entries of $\vec{x}(i, n)$, if $F \in \mathcal{P}_{f}(\mathbb{N}), k=\min F, r=\max F$, and $\varphi: F \rightarrow\{1,2, \ldots, \delta\}$, then $\sum_{t \in F} X_{\varphi(t), t} \subseteq A_{\varphi(k), \varphi(r)}$.

Proof. The proof is essentially the same as the proof of Theorem 3.1. At each point where one chose $x_{h, n} \in B_{h, n}^{\star}$, one instead chooses, using Theorem 3.4 and the fact that each $p_{i}$ is a member of $K(\beta \mathbb{N},+)$, some $\vec{x}_{h, n} \in\left(B_{h, n}^{\star}\right)^{m(n)}$ such that $M_{n} \vec{x}_{h, n}=\overrightarrow{0}$ and lets $X_{h, n}$ be the set of entries of $M_{n} \vec{x}_{h, n}$. The induction hypotheses then are:

(1) For $s \in\{1,2, \ldots, n\}$ and $h \in\{1,2, \ldots, \delta\}$

(a) $B_{h, s} \in p_{h}$ and if $s<n, B_{h, s+1} \subseteq B_{h, s}$ and

(b) $\vec{x}_{h, s} \in\left(B_{h, s}^{\star}\right)^{m(s)}, M_{s} \vec{x}_{h, s}=\overrightarrow{0}$, and $X_{h, s}$ is the set of entries of $\vec{x}_{h, s}$.

(2) If $\emptyset \neq F \subseteq\{1,2, \ldots, n\}, k=\min F, r=\max F$, and $\varphi: F \rightarrow\{1,2, \ldots, \delta\}$, then

(a) $\sum_{t \in F} X_{\varphi(t), t} \subseteq A_{\varphi(k), \varphi(r)}^{\star}$;

(b) if $r<n,(b, h, d, e) \in\{1,2, \ldots, k\}^{4}, z \in \sum_{t \in F} X_{\varphi(t), t}$, and $-z+$ $A_{\varphi(k), b}^{\star} \in p_{h}+p_{d}$, then $B_{h, r+1} \subseteq\left\{y \in \mathbb{N}:-(z+y)+A_{\varphi(k), b}^{\star} \in p_{e}+p_{d}\right\}$; and

\footnotetext{
${ }^{1}$ Furstenberg defined a subset $S$ of $\mathbb{N}$ to be central provided there exist a dynamical system $(X, T)$, a point $x \in X$, a uniformly recurrent point $y$ proximal to $x$, and a neighborhood $U$ of $y$ such that $S=\left\{n \in \mathbb{N}: T_{n}(x) \in U\right\}$.
} 
(c) if $r<n,(b, h) \in\{1,2, \ldots, k\}^{2}, z \in \sum_{t \in F} X_{\varphi(t), t}$, and $-z+A_{\varphi(k), b}^{\star} \in$ $p_{h}$, then $B_{h, r+1} \subseteq-z+A_{\varphi(k), b}^{\star}$.

The reader who wants to see more details should note that Theorem 3.5 is a corollary of Theorem 4.1, for which we will provide a detailed proof.

We obtain the promised partition corollary.

Corollary 3.6. Let $\delta \in \mathbb{N} \backslash\{1\}$. Let $\left\langle M_{n}\right\rangle_{n=1}^{\infty}$ enumerate the finite kernel partition regular matrices with rational coefficients and for $n \in \mathbb{N}$ let $m(n)$ be the number of columns of $M_{n}$. Let $\mathcal{R}$ be a finite partition of $\mathbb{N}$. For $(i, j) \in\{1,2, \ldots, \delta\}^{2}$, there exists $A_{i, j} \in \mathcal{R}$ such that for $i \in\{1,2, \ldots, \delta\}$ and $n \in \mathbb{N}$, there exists $\vec{x}(i, n) \in \mathbb{N}^{m(n)}$ such that $M_{n} \vec{x}(i, n)=\overrightarrow{0}$ and letting $X_{i, n}$ be the set of entries of $\vec{x}(i, n)$, if $F \in \mathcal{P}_{f}(\mathbb{N}), k=\min F, r=\max F$, and $\varphi: F \rightarrow\{1,2, \ldots, \delta\}$, then $\sum_{t \in F} X_{\varphi(t), t} \subseteq A_{\varphi(k), \varphi(r)}$. The partition $\mathcal{R}$ can be refined so that the sets $\left\{A_{i, j}:(i, j) \in\{1,2, \ldots, \delta\}^{2}\right\}$ are pairwise disjoint.

Proof. Pick $\left\langle p_{i}\right\rangle_{i=1}^{\delta}$ as in Theorem 3.5. For $(i, j) \in\{1,2, \ldots, \delta\}^{2}$, pick $A_{i, j} \in \mathcal{R}$ such that $A_{i, j} \in p_{i}+p_{j}$.

The final conclusion about the refinement of $\mathcal{R}$ follows from the fact that $\left\{p_{i}+p_{j}\right.$ : $\left.(i, j) \in\{1,2, \ldots, \delta\}^{2}\right\}$ is strongly discrete.

We now turn our attention to $(\mathbb{N}, \cdot)$. To say that a $u \times v$ matrix $M$ is kernel partition regular over $(\mathbb{N}, \cdot)$ means that whenever $\mathcal{R}$ is a finite partition of $\mathbb{N} \backslash\{1\}$ there exist $R \in \mathcal{R}$ and $\vec{x} \in R^{v}$ such that $\vec{x}^{M}=\overrightarrow{1}$ where entry $i$ of $\vec{x}^{M}$ is $\prod_{j=1}^{v} x_{j}^{m_{i, j}}$ and $m_{i, j}$ is the entry of $M$ in row $i$ and column $j$. As long as the entries of $M$ are integers, $M$ is kernel partition regular over $(\mathbb{N},+)$ if and only $M$ is kernel partition regular over $(\mathbb{N}, \cdot)$ by [8, Theorem 15.17]. But one looses the analogue of Theorem 3.4 .

For example the matrix $\left(\begin{array}{lll}2 & -2 & 1\end{array}\right)$ is kernel partition regular over $(\mathbb{N}, \cdot)$. That is, given any finite partition $\mathcal{R}$ of $\mathbb{N}$, there exist $R \in \mathcal{R}$ and $x_{1}, x_{2}, x_{3} \in R$ such that $x_{1}^{2} x_{2}^{-2} x_{3}=1$. But $\left\{x^{2}: x \in \mathbb{N}\right\}$ is not central in $(\mathbb{N}, \cdot)$. In fact it is an easy exercise to show that $A=\left\{x^{2}: x \in \mathbb{N}\right\}$ is not piecewise syndetic in $(\mathbb{N}, \cdot)$. That is, for every $G \in \mathcal{P}_{f}(\mathbb{N})$ there exists $F \in \mathcal{P}_{f}(\mathbb{N})$ such that for every $x \in \mathbb{N}$, $F x \backslash \bigcup_{t \in G} t^{-1} A \neq \emptyset$. By [8, Theorem 4.40], a set $A \subseteq S$ is piecewise syndetic if and only if $A \cap K(\beta S) \neq \emptyset$.

We do not have a reasonable characterization of those kernel partition regular matrices that have solutions in any multiplicatively central set, so we turn our attention to image partition regular matrices, where we do have a simple sufficient condition.

Definition 3.7. Let $(S,+)$ be an infinite commutative semigroup with identity 0 . Let $u, v \in \mathbb{N}$ and let $M$ be a $u \times v$ matrix with entries from $\omega$. The matrix $M$ is image partition regular over $S$ if and only if whenever $\mathcal{R}$ is a finite partition of $S \backslash\{0\}$, there exist $R \in \mathcal{R}$ and $\vec{x} \in(S \backslash\{0\})^{v}$ such that $M \vec{x} \in R^{u}$.

$$
\text { For example, the assertion that }\left(\begin{array}{ll}
1 & 0 \\
1 & 1 \\
1 & 2 \\
1 & 3
\end{array}\right) \text { is image partition regular over }(\mathbb{N},+)
$$

says that for any finite coloring of $\mathbb{N}$, there exist monochromatic length 4 arithmetic progressions. And the assertion that the same matrix is image partition regular 
over $(\mathbb{N}, \cdot)$ says that for any finite coloring of $\mathbb{N}$, there exist monochromatic length 4 geometric progressions.

Definition 3.8. Let $u, v \in \mathbb{N}$ and let $M$ be a $u \times v$ matrix. The matrix $M$ is a monic first entries matrix if and only if all entries of $M$ are from $\omega$, no row is equal to $\overrightarrow{0}$, and the leftmost nonzero entry of each row is 1 .

Theorem 3.9. Let $(S,+)$ be a commutative semigroup with identity 0 , let $u, v \in \mathbb{N}$, and let $M$ be a $u \times v$ monic first entries matrix. For every central subset $C$ of $S$, there exists $\vec{x} \in(S \backslash\{0\})^{v}$ such that $M \vec{x} \in C^{u}$.

Proof. This is a special case of [8, Theorem 15.5].

As we saw above a matrix guaranteeing image partition regularity of geometric prgressions in $\mathbb{N}$ is monic first entries so Theorem 1.5 follows from the following theorem. Recall that by Theorem 2.2, the sequence $\left\langle p_{i}\right\rangle_{i=1}^{\delta}$ hypothesized in this theorem exists.

Theorem 3.10. Let $\delta \in \mathbb{N} \backslash\{1\}$. Let $\left\langle M_{n}\right\rangle_{n=1}^{\infty}$ enumerate the monic first entries matrices and for $n \in \mathbb{N}$ let $m(n)$ be the number of columns of $M_{n}$. Let $\left\langle p_{i}\right\rangle_{i=1}^{\delta}$ be a sequence of distinct idempotents in $K(\beta \mathbb{N}, \cdot)$ such that for $(i, j) \in\{1,2, \ldots, \delta\}^{2}$, $p_{i} \cdot p_{j}$ is an idempotent and $\left\{p_{i} \cdot p_{j}:(i, j) \in\{1,2, \ldots, \delta\}^{2}\right\}$ are all distinct. Assume that for $(h, i, j) \in\{1,2, \ldots, \delta\}^{3}, p_{h} \cdot p_{i} \cdot p_{j}=p_{h} \cdot p_{j}$. For $(i, j) \in\{1,2, \ldots, \delta\}^{2}$, let $A_{i, j} \in p_{i} \cdot p_{j}$. For $i \in\{1,2, \ldots, \delta\}$ and $n \in \mathbb{N}$, there exists $\vec{x}(i, n) \in(\mathbb{N} \backslash\{1\})^{m(n)}$ such that, letting $X_{i, n}$ be the set of entries of $\vec{x}(i, n)^{M_{n}}$, if $F \in \mathcal{P}_{f}(\mathbb{N}), k=\min F$, $r=\max F$, and $\varphi: F \rightarrow\{1,2, \ldots, \delta\}$ then $\prod_{t \in F} X_{\varphi(t), t} \subseteq A_{\varphi(k), \varphi(r)}$.

Proof. This is the same as the proof of Theorem 3.5 except that, instead of choosing $\vec{x}_{i, n} \in B_{i, n}^{\star}{ }^{m(n)}$ such that $M_{n} \vec{x}_{i, n}=\overrightarrow{0}$ and letting $X_{i, n}$ be the set of entries of $M_{n} \vec{x}_{i, n}$, we choose $\vec{x}_{i, n} \in \mathbb{N}^{m(n)}$ such that all entries of $\vec{x}_{i, n}^{M_{n}}$ are in $B_{i, n}^{\star}$.

Of course we get the corresponding partition corollary.

Corollary 3.11. Let $\delta \in \mathbb{N} \backslash\{1\}$. Let $\left\langle M_{n}\right\rangle_{n=1}^{\infty}$ enumerate the monic first entries matrices and for $n \in \mathbb{N}$ let $m(n)$ be the number of columns of $M_{n}$. Lewt $\mathcal{R}$ be a finite partition of $\mathbb{N}$. For $(i, j) \in\{1,2, \ldots, \delta\}^{2}$, there exists $A_{i, j} \in \mathcal{R}$ such that for $i \in\{1,2, \ldots, \delta\}$ and $n \in \mathbb{N}$, there exists $\vec{x}(i, n) \in(\mathbb{N} \backslash\{1\})^{m(n)}$ such that, letting $X_{i, n}$ be the set of entries of $\vec{x}(i, n)^{M_{n}}$, if $F \in \mathcal{P}_{f}(\mathbb{N}), k=\min F, r=\max F$, and $\varphi: F \rightarrow\{1,2, \ldots, \delta\}$ then $\prod_{t \in F} X_{\varphi(t), t} \subseteq A_{\varphi(k), \varphi(r)}$. The partion $\mathcal{R}$ can be refined so that the sets $\left\{A_{i, j}:(i, j) \in\{1,2, \ldots, \delta\}^{2}\right\}$ are pairwise disjoint.

\section{Consequences of the existence of infinite ReCtangular semigroups}

We have in this section one main result which utilizes the existence of the sequence guaranteed by Corollary 2.6. As promised earlier, we will present the details of the proof. The basic ideas of the proof are the same as before. But the details are more complicated because, while we are dealing with infinitely many idempotents, the arguments depend at each stage on taking finite intersections of members of each of these.

Theorem 4.1. Let $\left\langle M_{n}\right\rangle_{n=1}^{\infty}$ enumerate the finite kernel partition regular matrices with rational coefficients and for $n \in \mathbb{N}$ let $m(n)$ be the number of columns of $M_{n}$. Let $\left\langle p_{i}\right\rangle_{i=1}^{\infty}$ be a sequence of distinct idempotents in $K(\beta \mathbb{N},+)$ such that for $(h, i, j) \in$ $\mathbb{N}^{3}, p_{i}+p_{j}$ is an idempotent, $p_{h}+p_{i}+p_{j}=p_{h}+p_{j}$, and $\left\{p_{i}+p_{j}:(i, j) \in \mathbb{N}^{2}\right\}$ is 
strongly discrete. For $(i, j) \in \mathbb{N}^{2}$, let $A_{i, j} \in p_{i}+p_{j}$ such that $\left\{A_{i, j}:(i, j) \in \mathbb{N}^{2}\right\}$ consists of pairwise disjoint sets. For $i \in \mathbb{N}$ and $n \in \mathbb{N}$ with $n \geq i$, there exists $\vec{x}(i, n) \in\left(A_{i, i}\right)^{m(n)}$ such that $M_{n} \vec{x}(i, n)=\overrightarrow{0}$ and letting $X_{i, n}$ be the set of entries of $\vec{x}(i, n)$, if $F \in \mathcal{P}_{f}(\mathbb{N}), k=\min F, r=\max F$, and $\varphi: F \rightarrow\{1,2, \ldots, k\}$, then $\sum_{t \in F} X_{\varphi(t), t} \subseteq A_{\varphi(k), \varphi(r)}$

Proof. We construct inductively for $n \in \mathbb{N}$ and $h \in\{1,2, \ldots, n\},\left\langle B_{h, s}\right\rangle_{s=h}^{n}$, $\left\langle\vec{x}_{h, s}\right\rangle_{s=h}^{n}$, and $\left\langle X_{h, s}\right\rangle_{s=h}^{n}$ satisfying the following induction hypotheses.

(1) For $h \in\{1,2, \ldots, n\}$ and $s \in\{h, h+1, \ldots, n\}$

(a) $B_{h, s} \in p_{h}$ and if $s<n, B_{h, s+1} \subseteq B_{h, s}$;

(b) $B_{h, s} \subseteq \bigcap_{i=1}^{s} \bigcap_{j=1}^{s}\left\{y \in \mathbb{N}:-y+A_{h, j}^{\star} \in p_{i}+p_{j}\right\}$;

(c) $B_{h, h} \subseteq A_{h, h}$; and

(d) $\vec{x}_{h, s} \in\left(B_{h, s}^{\star}\right)^{m(s)}, M_{s} \vec{x}_{h, s}=\overrightarrow{0}$, and $X_{h, s}$ is the set of entries of $\vec{x}_{h, s}$.

(2) If $\emptyset \neq F \subseteq\{1,2, \ldots, n\}, k=\min F, r=\max F$, and $\varphi: F \rightarrow\{1,2, \ldots, k\}$, then

(a) $\sum_{t \in F} X_{\varphi(t), t} \subseteq A_{\varphi(k), \varphi(r)}^{\star}$;

(b) if $r<n,(b, h, d, e) \in\{1,2, \ldots, k\}^{4}, z \in \sum_{t \in F} X_{\varphi(t), t}$, and $-z+$ $A_{\varphi(k), b}^{\star} \in p_{h}+p_{d}$, then $B_{h, r+1} \subseteq\left\{y \in \mathbb{N}:-(z+y)+A_{\varphi(k), b}^{\star} \in p_{e}+p_{d}\right\}$; and

(c) if $r<n,(b, h) \in\{1,2, \ldots, k\}^{2}, z \in \sum_{t \in F} X_{\varphi(t), t}$, and $-z+A_{\varphi(k), b}^{\star} \in$ $p_{h}$, then $B_{h, r+1} \subseteq-z+A_{\varphi(k), b}^{\star}$.

Let $B_{1,1}=A_{1,1}^{\star}$. Pick by Theorem 3.4, $\vec{x}_{1,1} \in\left(B_{1,1}^{\star}\right)^{m(1)}$ such that $M_{1} \vec{x}_{1,1}=\overrightarrow{0}$, and let $X_{1,1}$ be the set of entries of $\vec{x}_{1,1}$. Hypothesis (1) holds. For hypothesis (2), hypothesis (2)(a) says that $X_{1,1} \subseteq A_{1,1}^{\star}$, which is true.

Let $n \in \mathbb{N}$ and assume that the hypotheses hold for $n$. Let $B_{n+1, n+1}=$ $A_{n+1, n+1}^{\star} \cap \bigcap_{i=1}^{n+1} \bigcap_{j=1}^{n+1}\left\{y \in \mathbb{N}:-y+A_{n+1, j}^{\star} \in p_{i}+p_{j}\right\}$. Since for $i, j \in\{1,2, \ldots, n+$ $1\}, A_{n+1, j} \in p_{n+1}+p_{j}=p_{n+1}+p_{i}+p_{j},\left\{y \in \mathbb{N}:-y+A_{n+1, j}^{\star} \in p_{i}+p_{j}\right\} \in p_{n+1}$.

Now let $h \in\{1,2, \ldots, n\}$. We let $B_{h, n+1}$ be the intersection of

$$
B_{h, n}^{\star} \cap \bigcap_{i=1}^{n+1} \bigcap_{j=1}^{n+1}\left\{y \in \mathbb{N}:-y+A_{h, j}^{\star} \in p_{i}+p_{j}\right\}
$$

with sets of the following two forms. We note that in each case there are only finitely many sets being intersected. We also note that the intersection taken so far is in $p_{h}$.

(i) If $\emptyset \neq F \subseteq\{1,2, \ldots, n\}, k=\min F, r=\max F=n, \varphi: F \rightarrow\{1,2, \ldots, k\}$, and one has $(b, d, e) \in\{1,2, \ldots, k\}^{3}$ and $z \in \sum_{t \in F} X_{\varphi(t), t}$ such that $-z+$ $A_{\varphi(k), b}^{\star} \in p_{h}+p_{d}$, then intersect with $\left\{y \in \mathbb{N}:-(z+y)+A_{\varphi(k), b}^{\star} \in p_{e}+p_{d}\right\}$. Since $p_{h}+p_{d}=p_{h}+p_{e}+p_{d}$, these sets are in $p_{h}$.

(ii) If $\emptyset \neq F \subseteq\{1,2, \ldots, n\}, k=\min F, r=\max F=n, \varphi: F \rightarrow\{1,2, \ldots, k\}$, and one has $b \in\{1,2, \ldots, k\}$ and $z \in \sum_{t \in F} X_{\varphi(t), t}$ such that $-z+A_{\varphi(k), b} \in$ $p_{h}$, then intersect with $-z+A_{\varphi(k), b}^{\star}$.

Notice that $B_{h, n+1}$ is the intersection of finitely many members of $p_{h}$. Pick $\vec{x}_{h, n+1} \in\left(B_{h, n+1}^{\star}\right)^{m(n+1)}$ such that $M_{n+1} \vec{x}_{h, n+1}=\overrightarrow{0}$ and let $X_{h, n+1}$ be the set of entries of $\vec{x}_{h, n+1}$.

To verify hypothesis (1), let $h \in\{1,2, \ldots, n+1\}$ and let $s \in\{h, h+1, \ldots, n+1\}$. If $h \leq n$ and $s \leq n$, then hypothesis (1) holds by assumption. If $h=n+1$, in which 
case $s=n+1$, then hypothesis (1) holds by construction. If $h \leq n$ and $s=n+1$, again hypothesis (1) holds by construction.

To verify hypothesis $(2)$, let $\emptyset \neq F \subseteq\{1,2, \ldots, n+1\}$, let $k=\min F$, let $r=\max F$, and let $\varphi: F \rightarrow\{1,2, \ldots, k\}$. If $r<n$, then hypothesis (2) holds by assumption. Assume now that $r=n$. Then hypothesis (2)(a) holds by assumption. To verify (2)(b), assume we have $(b, h, d, e) \in\{1,2, \ldots, k\}^{4}$, and $z \in \sum_{t \in F} X_{\varphi(t), t}$ such that $-z+A_{\varphi(k), b}^{\star} \in p_{h}+p_{d}$. Then by item (i) in the construction, $B_{h, n+1} \subseteq$ $\left\{y \in \mathbb{N}:-(z+y)+A_{\varphi(k), b}^{\star} \in p_{e}+p_{d}\right\}$. To verify $(2)(\mathrm{c})$, assume we have $(b, h) \in$ $\{1,2, \ldots, k\}^{2}$ and $z \in \sum_{t \in F} X_{\varphi(t), t}$, such that $-z+A_{\varphi(k), b}^{\star} \in p_{h}$. Then by item (ii) in the construction, $B_{h, n+1} \subseteq-z+A_{\varphi(k), b}^{\star}$.

Now assume that $r=n+1$, so that (2)(b) and (2)(c) are vacuous. We shall verify (2)(a). Assume first that $|F|=1$, so that $F=\{n+1\}$ and $k=r=n+1$. Let $h=\varphi(n+1)$. Then $X_{\varphi(n+1), n+1} \subseteq B_{h, n+1}^{\star} \subseteq B_{h, h}^{\star} \subseteq A_{h, h}^{\star}$ as required.

Now assume that $|F| \geq 2$, let $G=F \backslash\{n+1\}$, and let $l=|G|$. Let $t_{1}, t_{2}, \ldots, t_{l}$ be the members of $G$ in order and let $t_{l+1}=n+1$. We shall show that

$$
\begin{aligned}
& \text { for } u \in\{1,2, \ldots, l\} \text { if } z \in \sum_{s=1}^{u} X_{\varphi\left(t_{s}\right), t_{s}} \text {, then } \\
& -z+A_{\varphi(k), \varphi(r)}^{\star} \in p_{\varphi\left(t_{u+1}\right)}+p_{\varphi(r)} .
\end{aligned}
$$

For $u=1, z \in X_{\varphi(k), k} \subseteq B_{\varphi(k), k}^{\star} \subseteq\left\{y \in \mathbb{N}:-y+A_{\varphi(k), \varphi(r)} \in p_{\varphi\left(t_{2}\right)}+p_{\varphi(r)}\right\}$ by hypothesis $(1)(b)$.

If $l=1$, then $(*)$ has been established. Assume that $l \geq 2, u \in\{2,3, \ldots, l\}$ and if $z \in \sum_{s=1}^{u-1} X_{\varphi\left(t_{s}\right), t_{s}}$, then $-z+A_{\varphi(k), \varphi(r)}^{\star} \in p_{\varphi\left(t_{u}\right)}+p_{\varphi(r)}$. Let $w \in \in \sum_{s=1}^{u} X_{\varphi\left(t_{s}\right), t_{s}}$ and pick $z \in \sum_{s=1}^{u-1} X_{\varphi\left(t_{s}\right), t_{s}}$ and $x \in X_{\varphi\left(t_{u}\right), t_{u}}$ such that $w=z+x$. Let $v=$ $t_{u-1}=\max \left\{t_{1}, t_{2}, \ldots t_{u-1}\right\}$. Then $v<n$ so by hypothesis $(2)(\mathrm{b})$ with $h=\varphi\left(t_{u}\right)$, $e=\varphi\left(t_{u+1}\right)$ and $d=b=\varphi(r),-z+A_{\varphi(k), \varphi(r)} \in p_{\varphi\left(t_{u}\right)}+p_{\varphi(r)}$ so $B_{\varphi\left(t_{u}\right), v+1} \subseteq$ $\left\{y \in \mathbb{N}:-(z+y)+A_{\varphi(k), \varphi(r)}^{\star} \in p_{\varphi\left(t_{u+1}\right)}+p_{\varphi(r)}\right.$. Then $x \in X_{\varphi\left(t_{u}\right), t_{u}} \subseteq B_{\varphi\left(t_{u}\right), t_{u}}^{\star} \subseteq$ $B_{\varphi\left(t_{u}\right), v+1}$ so $-w+A_{\varphi(k), \varphi(r)}^{\star} \in p_{\varphi\left(t_{u+1}\right)}+p_{\varphi(r)}$. Thus $(*)$ has been established. In particular if $z \in \sum_{s=1}^{l} X_{\varphi\left(t_{s}\right), t_{s}}$, then $-z+A_{\varphi(k), \varphi(r)}^{\star} \in p_{\varphi\left(t_{l+1}\right)}+p_{\varphi(r)}=p_{\varphi(r)}$ since $t_{l+1}=n+1=r$. That is, if $z \in \sum_{t \in G} X_{\varphi(t), t}$, then $-z+A_{\varphi(k), \varphi(r)}^{\star} \in p_{\varphi(r)}$.

Let $v=\max G$. Let $w \in \sum_{t \in F} X_{\varphi(t), t}$ and pick $z \in \sum_{t \in G} X_{\varphi(t), t}$ and $x \in$ $X_{\varphi(r), r}$ such that $w=z+x$. Then $B_{\varphi(r), v+1} \subseteq-z+A_{\varphi(k), \varphi(r)}^{\star}$ by item (ii) in the construction if $v=n$ and by hypothesis (2)(c) applied to $G$ with $b=h=\varphi(r)$ if $v<n$. Then $x \in X_{\varphi(r), r} \subseteq B_{\varphi(r), v+1}$ so $w \in A_{\varphi(k), \varphi(r)}^{\star}$.

Corollary 4.2. Let $\left\langle M_{n}\right\rangle_{n=1}^{\infty}$ enumerate the finite kernel partition regular matrices with rational coefficients and for $n \in \mathbb{N}$ let $m(n)$ be the number of columns of $M_{n}$. Let $\mathcal{R}$ be a finite partition of $\mathbb{N}$. For $i \in \mathbb{N}$, there exists $B_{i} \in \mathcal{R}$ and for $(i, j) \in \mathbb{N}^{2}$, there exists $A_{i, j} \in \mathcal{R}$ such that for $i \in \mathbb{N}$ and $n \in \mathbb{N}$, there exists $\vec{x}(i, n) \in \mathbb{N}^{m(n)}$ such that $M_{n} \vec{x}(i, n)=\overrightarrow{0}$ and letting $X_{i, n}$ be the set of entries of $\vec{x}(i, n)$,

(1) for $i \in \mathbb{N}$ and $F \in \mathcal{P}_{f}(\mathbb{N})$ with $\min F \geq i, \sum_{t \in F} X_{i, t} \subseteq B_{i}$ and

(2) if $l \in \mathbb{N}, F_{1}, F_{2}, \ldots, F_{l} \in \mathcal{P}_{f}(\mathbb{N})$, for $s \in\{1,2, \ldots, l-1\}$, if any, $\max F_{s}<$ $\min F_{s+1}, \varphi:\{1,2, \ldots, l\} \rightarrow \mathbb{N}$, and $\min F_{1} \geq \max \varphi[\{1,2, \ldots, l\}]$, then $\sum_{s=1}^{l} \sum_{t \in F_{s}} X_{i, t} \subseteq A_{\varphi(1), \varphi(l)}$. 
In Corollary 4.2 we cannot add the conclusion that the partition can be refined to make the chosen sets pairwise disjoint, for the simple reason that one cannot get infinitely many pairwise disjoint subsets of a finite partition.

\section{REFERENCES}

[1] Vitaly Bergelson, Hillel Furstenberg, Neil Hindman, and Yitzhak Katznelson, An algebraic proof of van der Waerden's theorem, Enseign. Math. (2) 35 (1989), no. 3-4, 209-215. MR:1039944

[2] Vitaly Bergelson and Neil Hindman, A combinatorially large cell of a partition of $\mathbf{N}$, J. Combin. Theory Ser. A 48 (1988), no. 1, 39-52, DOI 10.1016/0097-3165(88)90073-8. MR938856

[3] Vitaly Bergelson and Neil Hindman, Nonmetrizable topological dynamics and Ramsey theory, Trans. Amer. Math. Soc. 320 (1990), no. 1, 293-320, DOI 10.2307/2001762. MR982232

[4] Dennis Davenport, Neil Hindman, Imre Leader, and Dona Strauss, Continuous homomorphisms on $\beta \mathbf{N}$ and Ramsey theory, New York J. Math. 6 (2000), 73-86. MR.1750426

[5] Walter Deuber and Neil Hindman, Partitions and sums of $(m, p, c)$-sets, J. Combin. Theory Ser. A 45 (1987), no. 2, 300-302, DOI 10.1016/0097-3165(87)90020-3. MR894824

[6] H. Furstenberg, Recurrence in ergodic theory and combinatorial number theory, Princeton University Press, Princeton, N.J., 1981. M. B. Porter Lectures. MR603625

[7] Neil Hindman, Finite sums from sequences within cells of a partition of $N$, J. Combinatorial Theory Ser. A 17 (1974), 1-11, DOI 10.1016/0097-3165(74)90023-5. MR349574

[8] Neil Hindman and Dona Strauss, Algebra in the Stone-Čech compactification, De Gruyter Textbook, Walter de Gruyter \& Co., Berlin, 2012. Theory and applications; Second revised and extended edition [of MR1642231]. MR2893605

[9] Neil Hindman, Dona Strauss, and Yevhen Zelenyuk, Large rectangular semigroups in StoneČech compactifications, Trans. Amer. Math. Soc. 355 (2003), no. 7, 2795-2812, DOI 10.1090/S0002-9947-03-03276-8. MR.1975400

[10] Richard Rado, Studien zur Kombinatorik (German), Math. Z. 36 (1933), no. 1, 424-470, DOI 10.1007/BF01188632. MR.1545354

[11] Hong-ting Shi and Hong-wei Yang, Nonmetrizable topological dynamical characterization of central sets, Fund. Math. 150 (1996), no. 1, 1-9, DOI 10.4064/fm-150-1-1-9. MR1387952

[12] B. van der Waerden, Beweis einer Baudetschen Vermutung, Nieuw Arch. Wiskunde 19 (1927), 212-216.

[13] Yevhen Zelenyuk, Elements of order 2 in $\beta \mathbb{N}$, Fund. Math. 252 (2021), no. 3, 355-359, DOI 10.4064/fm902-5-2020. MR4178872

[14] Yevhen Zelenyuk, Finite semigroups in $\beta \mathbb{N}$ and Ramsey theory, Bull. Lond. Math. Soc. 53 (2021), no. 3, 710-722, DOI 10.1112/blms.12453. MR 4275083

Department of Mathematics, Ohio State University, Columbus, Ohio 43210

Email address: vitaly@math.ohio-state.edu

Department of Mathematics, Howard University, Washington, DC 20059

Email address: nhindman@aol.com 